\title{
Glide symmetry to prevent the lowest stopband of printed corrugated transmission lines
}

\author{
P. Padilla, L.F. Herrán, A. Tamayo-Domínguez, J. F. Valenzuela-Valdés and O. Quevedo- \\ Teruel
}

\begin{abstract}
In this letter, we demonstrate that the dispersion properties of printed double-sided parallel-strip lines can be controlled by using glide symmetry. Glide symmetry is introduced in with corrugations in both strips of a double-sided line. We demonstrate that glide symmetry eliminates the stopband between first and second propagating modes and yields to a higher propagation constant, preserving its linearity and the broadband nature of the underlying guiding technology. Thus, the glide-symmetric double-sided line can be designed to possess a high equivalent refractive index in an ultra-wide range of frequencies. These exceptional properties have been numerically and experimentally validated. Finally, we demonstrate the possibilities of this technology with a specific design, a glidesymmetric double-sided parallel-strip line with filtering properties. Potential applications are low-dispersive leaky wave antennas, electromechanical tunable phase shifters and filters.
\end{abstract}

Index Terms - Higher symmetries, Microwave printed circuits, Metamaterials.

\section{INTRODUCTION}

The extraordinary properties of higher-symmetric structures were identified in the $60 \mathrm{~s}$ and 70 s for onedimensional structures [1]. However, it was only recently, with the advent of metamaterials, when higher symmetries found their niche of application, especially for high frequency antennas and integrated circuits. For example, higher symmetries have been utilized for producing low-dispersive lens antennas [2], costeffective gap waveguide technology [3], low-cost contactless flanges [4], low-dispersive transmission lines [5].

There are two known types of higher symmetries: glide and twist. A periodic structure possesses glide symmetry if its unit cell is generated with a mirroring and a translation of half of the period [1]. On the contrary, twist symmetry is originated with a translation and an angular rotation [6]. To produce an operational difference with respect to conventional periodic structures, the constituent sub-cells of a higher-symmetric unit cell must be strongly coupled, which implies short distances between sub-cells. The recent interest for practical applications has elevated the number of studies on fast analyses based on either circuit models [7] or mode-matching techniques [8].

Here, we present a new kind of glide-symmetric structure and we evaluate its potential for lowdispersive propagation and filtering properties. We apply this glide symmetry to the double-sided parallel-strip line, which has been widely used in the design passive components, antennas, and EBG structures.

\section{GLIDE-SYMMETRIC DOUBLE-SIDED PARALLEL-STRIP LINE}

The structure is a double-sided parallel-strip line with glidesymmetric planar corrugations as depicted in Fig. 1.a. Both printed lines are made of copper and placed on each side of a dielectric substrate. The double-sided strips include periodic metallic corrugations with a period $p$ along the line axis (x axis), which are misaligned $p / 2$ in this axis, so the structure possesses glide symmetry. The glide symmetry plane is normal to the $\mathrm{z}$ axis, located in the middle of the substrate.

In our case study, the baseline double-sided parallel-strip line is printed over an Arlon $25 \mathrm{~N}$ substrate and is designed to have a characteristic impedance of $50 \mathrm{Ohm}$. Notice that, depending on the corrugation widths ( $w_{1}$ and $w_{2}$ ), overlapping between the corrugations at both sides of the line is possible.

The proposed structure was simulated with the eigenmode analysis of CST Microwave Studio for the dimensions: $p=8 \mathrm{~mm}, w_{l}=w_{2}=p / 1.5$, sep $=p / 2, h_{d i e}=0.762 \mathrm{~mm}$ and $g=2.3 \mathrm{~mm}$. The results for different lengths of the corrugations $\left(h_{1}\right.$ and $\left.h_{2}\right)$ are represented in Fig 1.b. Glide symmetry introduces two effects in the structure. First, it decreases the frequency dependence of the first propagating mode, whose propagation constant becomes almost linear with frequency. Second, it 
eliminates the closed stopband between first and second propagation modes. For the sake of clarity, we have included the vacuum lightline and the one for the analytic effective permittivity of the double-sided parallel-strip line.

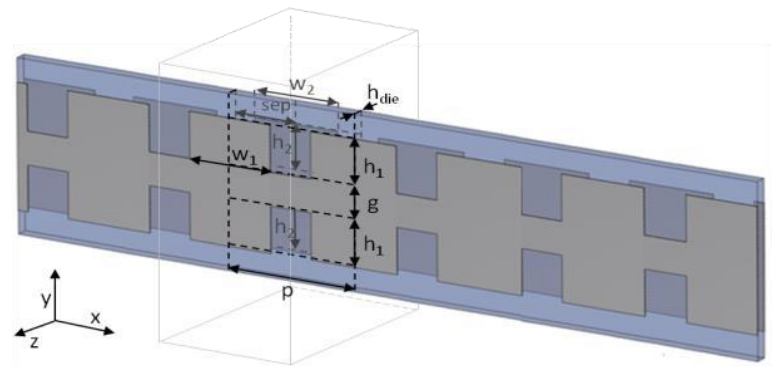

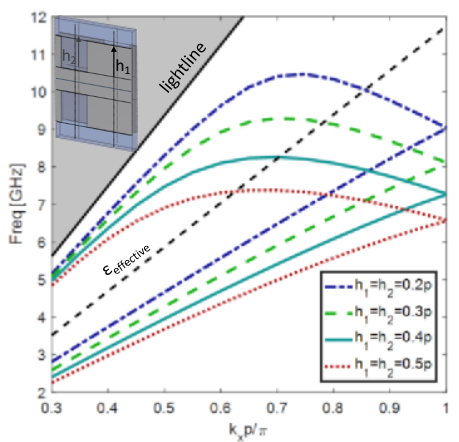

b)

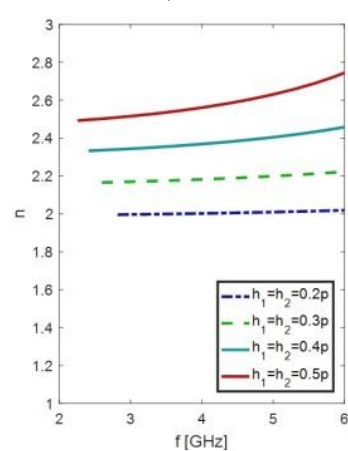

c)

Fig. 1. Planar double-sided line with glide-symmetric corrugations: a) Structure and its constituent parameters, b) simulated dispersion diagram for the first two modes, when modifying the corrugations lengths $\left(h_{l}=h_{2}\right)$, when the dimensions are: $p=8 \mathrm{~mm}, w_{l}=w_{2}=p / 1.5$, $\mathrm{sep}=p / 2, h_{\text {die }}=0.762 \mathrm{~mm}$ and $g=2.3 \mathrm{~mm}, \mathrm{c}$ ) equivalent refractive index for the first mode.

The variation in length introduces a variation in the frequency at which the modes join and a variation in the propagation constant slope. Longer lengths increase the propagation constant and therefore, the equivalent refractive index of the double-sided parallel-strip line, while maintaining a broadband response. Same as the length of the corrugations, the width plays an important role in the definition of the propagation constant. The effect of the variations in the width of the corrugations $\left(w_{l}=w_{2}\right)$ is presented in Fig. 2.a.

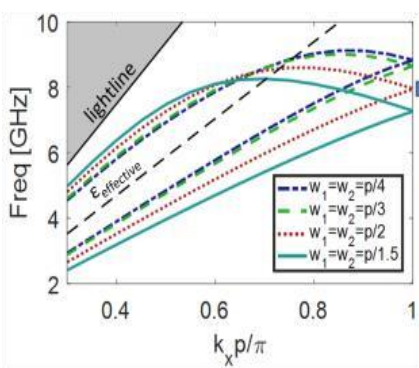

a)

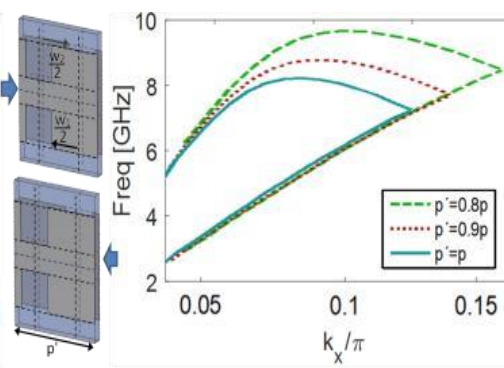

b)

Fig. 2. Simulated dispersion diagram of the glide-symmetric unit cell, when a) modifying the corrugations width $\left(w_{1}\right.$ and $\left.w_{2}\right)$, preserving glide symmetry $\left(w_{l}=w_{2}\right)$, and b) when reducing the cell periodicity $\left(p^{\prime}\right)$. Reference dimensions are: $p=8 \mathrm{~mm}, w_{l}=w_{2}=p / 1.5$, $h_{1}=h_{2}=0.4 p$, sep $=p / 2, h_{\text {die }}=0.762 \mathrm{~mm}$ and $g=2.3 \mathrm{~mm}$.

Wider values of the width increase the equivalent refractive index of the double-sided line, while the linearity of the first mode is preserved. In Fig. 2.b, we represent the effect of changing the periodicity ( $\left.p^{\prime}\right)$, where the width and length of the corrugations remain constant. Reducing the periodicity permits the propagation of the first mode at higher frequencies, while the propagation constant remains the same for the lower frequencies. Notice that in this specific case, to provide an adequate comparison, the abscissa axis is not normalized with respect to the periodicity. 


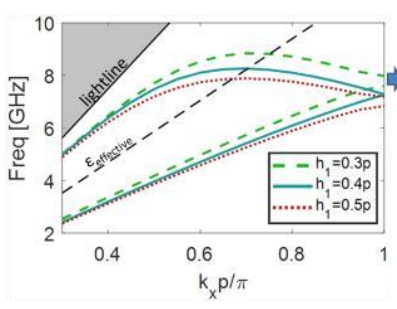

a)

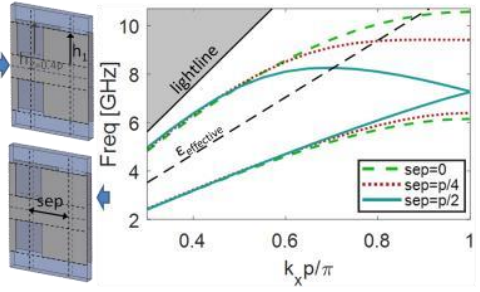

b)

Fig. 3. Effects of breaking the glide symmetry in the propagation constant: a) Varying only $h_{l}$, and fixed $h_{2}=0.4 p$, and sep=0.5p, b) varying the separation between corrugations, sep, for $h_{l}=h_{2}=0.4 p$. The rest of dimensions are the baseline ones: $p=8 \mathrm{~mm}, w_{l}=w_{2}=p / 1.5$, $h_{\text {die }}=0.762 \mathrm{~mm}$ and $g=2.3 \mathrm{~mm}$.

Additionally, the structure response can also be modified by breaking the glide symmetry conditions. This can be made either by independently varying $h_{1}$ and $h_{2}, w_{1}$ and $w_{2}$, or with sep $\neq 0.5 p$. The rest of the work is referred to the following dimensions: $h_{l}=h_{2}=0.4 p, w_{l}=w_{2}=p / 1.5$ and sep=0.5p. In Fig. 3, we illustrate the simulated effect of breaking the glide symmetry, modifying the length and the separation (sep).

Figure 3.a shows the effect when modifying one of the lengths $\left(h_{1}\right)$, while the other one is maintained constant $\left(h_{2}=0.4 p\right)$. The rupture of the glide symmetry introduces a stopband between the first and second mode. The stopband location depends on the electrical length of the corrugations.

Figure 3.b depicts the effect when the separation between top and bottom corrugations is not $p / 2$. Particularly, the dispersion diagram for separation values $s e p=p / 4$ and 0 are compared with the glide-symmetric case, $p / 2$. The case of $\mathrm{sep}=0$ is the one that provides the widest closed stopband.

Both plots in Fig. 3 reveal that the first mode is almost linear with respect to the frequency for the glide configuration. If this symmetry is broken, the propagation constant for the first mode becomes more dispersive, and a stopband between the first two modes appears.

\section{EXPERIMENTAL VALIDATION}

To validate our simulated results of the printed glidesymmetric double-sided line, an experimental demonstration was conducted. Two prototypes were manufactured as shown in Fig 4.a and Fig 4.b and the transversal electric field over the desired area (dashed rectangle) at $7 \mathrm{GHz}$ was measured with a probe at $100 \mu \mathrm{m}$ above the circuit. The first prototype is in accordance to the dimensions for the results in Fig. 3.b with sep=p/2 (glide), while the second one corresponds with sep $=0$ (non-glide). Both prototypes were designed with a guiding transition at left and right sides for matching purposes, fed with edge-launch SMA connector at each port.
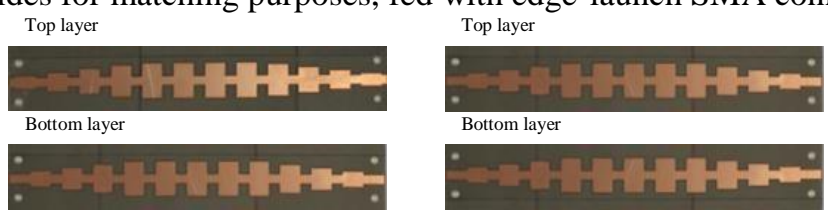

a)

b)

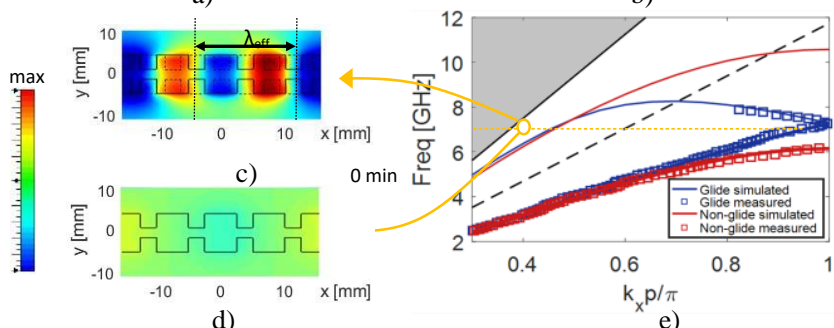

Fig. 4. Validation through prototypes: a) Glide-symmetric prototype, same dimensions as in Fig. 3.b and sep=p/2, b) nonglide prototype, same dimensions as in Fig. 3.b and $\mathrm{sep}=0$, c) measured electric field at $7 \mathrm{GHz}$ of the glide-symmetric prototype, d) Measured electric field at $7 \mathrm{GHz}$ of the nonglide prototype, e) Comparison of simulated and measured dispersion diagrams for both designs. The measured electric field in c) shows the effective wavelength $\left(\lambda_{\text {eff }}\right)$ of the glide structure.

Figures 4.c and 4.d show the measured field distributions. Fig. 4.c demonstrates that the glide structure allows the propagation of the first mode. On the contrary, the field distribution in Fig. 
4.d shows that the non-glide structure has practically no propagation, due to the existence of a closed stopband, and only residual fields are excited above the structure with almost free space wavelength. Additionally, Fig. 4.e shows the measured dispersion diagrams for both designs. These diagrams were obtained with the phase value of the $S_{21}$ (dashed white box), once the effect of the transitions is eliminated. The measurements demonstrate an excellent agreement with the simulated values shown in Fig. 3.b.

After the validation of the dispersion properties, we propose a possible practical use of this structure for filtering purposes. To validate this concept, we have defined a guiding doublesided parallel-strip structure with a progressive change to break the symmetry. The left port of the design possesses the dimensions of the structure in Fig. 3.b and sep=0.5p. Along the guiding structure, the misalignment distance between top and bottom layers, sep, is progressively modified from $0.5 p$ (glide) to 0 (non-glide).

Therefore, the guiding structure presents a closed stopband that is progressively increasing along the line. Depending on the chosen frequency, the line is able to either permit or progressively stop the field propagation along the structure. Figure 5.a shows the model of this device, and Fig. 5.b a photograph of the prototype. Experimental results of the transversal electric field distribution measured above the circuit are represented in Fig. 5.c. The plotted field frequencies are in the passband frequency range at the glide port (left side) but in the stopband frequency range for the non-glide port (right side). Notice that the pass/stop range is obtained from Fig. 3.b. The measurements provide a field propagation that matches the expected response: for $7.1 \mathrm{GHz}$ the field is stopped within a shorter distance than for $6.3 \mathrm{GHz}$.

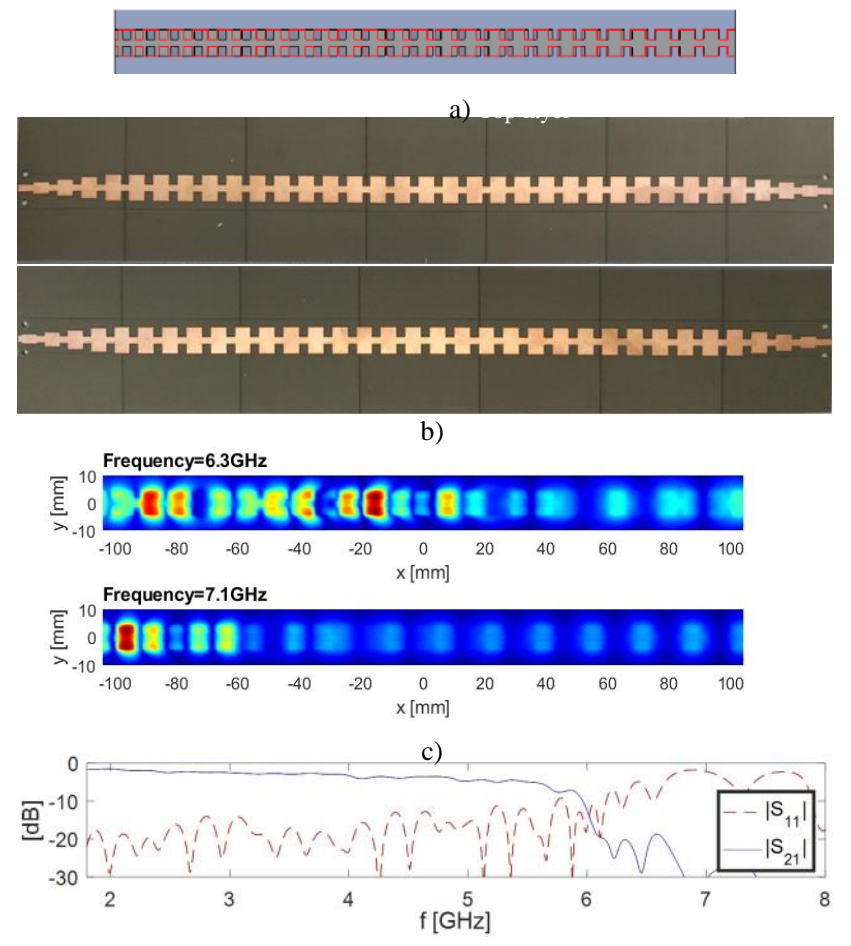

d)

Fig. 5. Glide-to-non-glide filtering structure: a) filter model (upper layer with black contour and bottom layer with red contour), b) prototype, upper and bottom layers, where the measured area is inside the dashed rectangle, c) measured field distribution over the upper layer at frequencies $6.3 \mathrm{GHz}$ and $7.1 \mathrm{GHz}$, and d) $\left|\mathrm{S}_{11}\right|$ and $\left|\mathrm{S}_{21}\right|$ of the filtering structure.

Corrugated double-sided lines have been previously used to design filters and other components $[9,10]$. In these cases, asymmetry offers higher impedance and more flexibility for the design. In our particular case, we impose glide symmetry.

\section{CONCLUSION}

In conclusion, here, we have demonstrated that a conventional double-sided parallel-strip line can benefit from the introduction of higher symmetries to vary its guiding properties. With glidesymmetric periodicities added through printed transversal lines (corrugations), the propagation constant can be tuned preserving the linearity (broadband response) of the underlying guiding technology. The corrugations make the propagation constant larger, while the glide symmetry 
guarantees the preservation of the linearity and the absence of a stopband between the first two modes. The modification of the corrugation dimensions (length and width) and periodicity, preserving the glide symmetry, can be used to modify the line response with no stopband between the first and second modes. By breaking the glide symmetry, the structure response has a closed stopband, which can be used to produce filtering effects at chosen frequency ranges. We demonstrate these filtering properties through the design and measurement of a prototype, varying the misalignment between layers. The prototype is progressively varied from glide symmetry to parallel corrugated lines, so that depending on the chosen frequency, the line is able to either permit or progressively stop the field propagation along the line.

\section{REFERENCES}

[1] A. Hessel, M.-H. Chen, R.-C. Li, and A.-A. Oliner, "Propagation in periodically loaded waveguides with higher symmetries", Proc. IEEE, vol. 61, no. 2, pp. 183-195, 1973.

[2] O. Quevedo-Teruel, M. Ebrahimpouri, and M. Ng Mou Kehn,

"Ultrawideband metasurface lenses based on off-shifted opposite layers", IEEE Antennas Wirel. Propag. Lett., vol. 15, pp. 484-487, 2016

[3] M. Ebrahimpouri, E. Rajo-Iglesias, Z. Sipus, and O. Quevedo-Teruel, "Cost-effective gap waveguide technology based on glide-symmetric holey EBG structures”, IEEE Trans. Microw. Theory Tech., vol. 66, no. 2, pp. 927-934 Feb. 2018.

[4] M. Ebrahimpouri, A. Algaba Brazalez, L. Manholm, O. QuevedoTeruel, "Using glide-symmetric holes to reduce leakage between waveguide flanges", IEEE Microw. Wirel. Compon. Lett. Online 2018.

[5] M. Camacho, R.-C. Mitchell-Thomas, A.-P. Hibbins, J.-R. Sambles, and O. Quevedo-Teruel, "Mimicking glide symmetry dispersion with coupled slot metasurfaces," Appl. Phy. Lett., vol. 111, no. 12, 2017.

[6] O. Dahlberg, R.-C. Mitchell-Thomas, and O. Quevedo-Teruel, "Reducing the dispersion of periodic structures with twist and polar glide symmetries”, Sci. Rep., vol. 7, no. 1, pp. 10136, 2017.

[7] G. Valerio, Z. Sipus, A. Grbic, and O. Quevedo-Teruel, “Accurate equivalent-circuit descriptions of thin glidesymmetric corrugated metasurfaces”, IEEE Trans. Antennas Propag., vol. 65, no. 5, pp. 26952700, May 2017.

[8] F. Ghasemifard, M. Norgren, and O. Quevedo-Teruel, "Dispersion analysis of two dimensional glide-symmetric corrugated metasurfaces using mode-matching technique", IEEE Microw. Wirel. Compon. Lett. vol. 28, no. 1, pp. 1-3, Jan. 2018.

[9] S.G. Kim and K. Chang, "Ultrawide-band transitions and new microwave components using double-sided parallelstrip lines,” IEEE Trans. Microw. Theory Tech., vol. 52, no. 9, pp. 2148-2152, Sep. 2004.

[10] S. Sun and L. Zhu, "Stopband-enhanced and size-miniaturized low-pass filters using high-impedance property of offset finite-ground microstrip line," IEEE Trans. Microw. Theory Tech., vol. 53, no. 9, pp. 2844-2850, Sep. 2005.

Manuscript received Jan, 2018.

This work has been supported by the Spanish Program of Research, Development and Innovation (ref. TIN2016-75097-P, UNGR15-CE-3311) and the Salvador de Madariaga Program (ref. PRX17/00100, PRX17/00630)

P. Padilla and J.F. Valenzuela-Valdés with the Department of Signal, Theory Telematics and Communications, Universidad de Granada, Granada, Spain (e-mail: pablopadilla@ugr.es).

L.F. Herrán is with the Department of Electrical Engineering, University of Oviedo, Gijón, Spain

A. Tamayo-Domínguez is with the Department of Signal, Systems and Radiocommunications, Technical University of Madrid, Madrid, Spain

O. Quevedo-Teruel is with the Department of Electromagnetic Engineering, KTH Royal Institute of Technology, Stockholm, Sweden 